\title{
Management of BU-HIV Co-infection
}

\author{
Daniel P. O'Brien, Vanessa Christinet, and Nathan Ford
}

\section{$1 \quad$ Epidemiology}

In many Buruli ulcer (BU) endemic countries worldwide there is also a high HIV prevalence. This is especially the case in Africa with adult HIV prevalence rates between 1 and 5\% in BU endemic countries. Evidence from Benin, Cameroon, Ghana and Gabon suggest that HIV may increase the risk of BU [1-5]. For example, in the Médecins Sans Frontières programme in Akonolinga, Cameroon, the prevalence of HIV was approximately 3-6 times higher in BU treated patients compared to the regional estimated HIV prevalence (37\% vs $8 \%$ in women; $20 \%$ vs $5 \%$ in men; and $4 \%$ vs $0.7 \%$ in children) [2]. Likewise in Benin, in one region patients with BU were eight times more likely to have HIV infection than those without BU (2.6\% vs $0.3 \%$ ) [3], and in another region adults with BU were five times more likely to have HIV compared to the regional estimated HIV prevalence $(5.0 \%$ vs $1.1 \%$ ) [4]. In Ghana HIV prevalence was 4-5 times higher in BU patients than those without BU (5\% vs $0.9 \%$ in one study and $8.2 \%$ vs $2.5 \%$ in another study) $[1,6]$. Therefore, there is a significant potential for the two infections to overlap in the same individual and HIV needs to be considered in all BU patients in settings with high background HIV prevalence.

D. P. O'Brien $(\varangle)$

Department of Infectious Diseases, Barwon Health, Geelong, VIC, Australia

Department of Medicine and Infectious Diseases, Royal Melbourne Hospital, University of Melbourne, Melbourne, VIC, Australia

e-mail: Danielo@BarwonHealth.org.au

V. Christinet

Centre International de Recherches, d'Enseignements et de Soins en milieu tropical (CIRES),

Akonolinga, Cameroon

N. Ford

HIV Department, World Health Organization, Geneva, Switzerland 


\section{Clinical Effects of BU-HIV Co-Infection}

HIV appears to affect the clinical presentation and severity of BU disease with a reported increased incidence of multiple, larger and ulcerated BU lesions in HIVinfected individuals (Fig. 1) [1, 2, 4, 5]. In Cameroon BU-HIV co-infected patients have a median lesion size of $5.5 \mathrm{~cm}$ (equivalent to WHO category 2) and up to $24 \%$ have multiple BU lesions (equivalent to WHO category 3) [2]. This results in increased patient morbidity as well as more time and resources required to treat lesions, and increased rates of long-term disability. Additionally it appears that the presence and severity of BU may reflect the level of underlying immune suppression in an HIV-infected person. In Akonolinga, Cameroon, $80 \%$ of patients with category 2 or $3 \mathrm{BU}$ lesions had a CD4 count $\leq 500$ cells $/ \mathrm{mm}^{3}$ compared to $55 \%$ of those with category 1 lesions, and the main lesion size was significantly increased with decreasing CD4 cell counts [2].

BU-HIV co-infected patients often present with severe immunosuppression and thus in urgent need of antiretroviral therapy (ART). In Akonolinga, 22\% of patients diagnosed with BU were classified as having advanced HIV disease (defined as CD4 counts $\leq 200$ cells $/ \mathrm{mm}^{3}$ ) and $48 \%$ had CD4 counts between 200 and 500 cells $/ \mathrm{mm}^{3}$ [2]. In this programme, mortality in BU-HIV co-infected patients treated for BU without ART was significantly higher than for HIV non-infected BU patients (11\% vs $1 \%$, $p<0.001)$. This is despite median CD4 cell counts at baseline among the 8 deceased HIV-infected patients being only moderately reduced at 229 cells $/ \mathrm{mm}^{3}$, levels usually not associated with such a high mortality rate. Death occurred early with a median time to death post BU diagnosis of 41.5 days and none had received ART. This suggests that a delay in ART initiation until after the completion of the recommended 8 weeks BU antibiotic treatment may adversely affect mortality rates [2]. In Ghana,

Fig. 1 Bilateral severe BU lesions on the lower limbs of an HIV co-infected patient

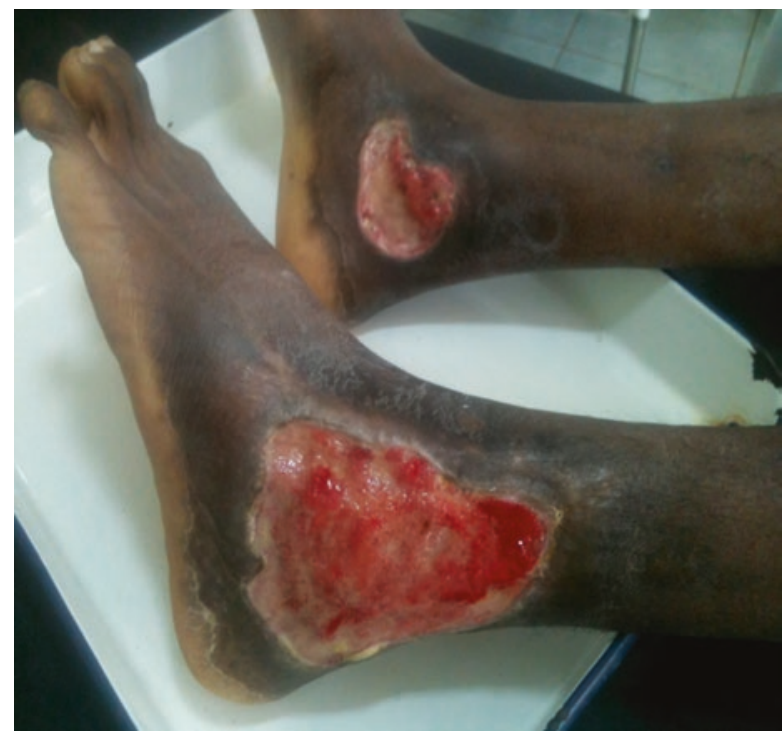


during the management of BU lesions, two of four patients $(50 \%)$ who did not start ART died compared with none of three $(0 \%)$ who commenced ART [1]. In Gabon, all nine $(100 \%)$ patients with known HIV infection died during the treatment of BU, although data regarding their ART status was not reported [5]. There are also several case reports describing deaths in BU-HIV patients not commenced on ART [7-9].

\section{Diagnosis}

It is not known whether co-infection with HIV affects the sensitivity or specificity of BU diagnostic tests, but it appears unlikely. Therefore recommended methods of BU diagnosis are the same as for HIV negative patients. This includes mainly IS2404 PCR testing of specimens, and microscopic detection of acid fast bacilli in stained smears from wound specimens [10]. As BU patients appear to represent a sentinel group with a higher prevalence of HIV, and knowing that HIV status will affect treatment options and may influence mortality and BU outcomes, it is recommended that all BU patients presenting in settings with a high prevalence of HIV have their HIV status determined by offering quality provider-initiated HIV testing and counseling [11].

\section{Management of BU-HIV Co-Infection}

The management of BU-HIV co-infection presents a complex and challenging situation for clinicians involved in patient care. Effective management requires attention to the treatment of each infection, but also consideration of the potential interactions between the treatments.

\subsection{BU Treatment}

If the patient is not already on ART, BU treatment should be commenced first, with combination antibiotic treatment. This is recommended prior to commencing ART for HIV to minimize pill burden and avoid drug interactions and side effects in the early stages of BU treatment, to allow the time needed for patient preparation for ART, and to follow the usual principle of HIV care to treat and stabilize any coinfections prior to commencing ART [12].

In 2017 the WHO Technical Advisory Group on BU decided that the WHO recommendation for BU treatment in Africa should be changed to rifampicin $10 \mathrm{mg} / \mathrm{kg}$ daily up to a maximum of $600 \mathrm{mg} /$ day plus clarithromycin $7.5 \mathrm{mg} / \mathrm{kg}$ twice daily (up to a maximum of $1000 \mathrm{mg}$ daily). However, due to potential drug interactions this combination should be used with caution. An alternative is rifampicin $10 \mathrm{mg} / \mathrm{kg}$ daily up to a maximum of $600 \mathrm{mg} /$ day plus moxifloxacin $400 \mathrm{mg}$ daily. The use of the combination of rifampicin and streptomycin is no longer recommended due to unacceptable toxicity levels such as renal dysfunction and ototoxicity, drug 
interactions with tenofovir, as well as the negative effects on adherence of daily painful injections. There is no evidence that the duration of BU antibiotic treatment needs to be prolonged beyond the standard recommended 8-week course for BU-HIV co-infected patients [13].

\subsection{HIV Treatment}

As currently recommended for all HIV-infected individuals, a CD4 cell count should be determined for all BU-HIV positive patients to assess the level of HIVassociated immune suppression. If the CD4 cell count is equal to or $<350$ cells $/ \mathrm{mm}^{3}$ then prophylactic cotrimoxazole ( $960 \mathrm{mg}$ tablet daily) should be commenced immediately to reduce mortality, morbidity and HIV disease progression [14]. If a CD4 cell count is not available and the patients have advanced HIV disease (WHO clinical stages 3 or 4) they should likewise receive cotrimoxazole prophylaxis. In areas with high prevalence of malaria and/or bacterial infections, cotrimoxazole should be commenced regardless of CD4 cell count in all BU-HIV-infected patients and continued for life. Patients with advanced HIV disease are at risk of a range of other opportunistic infections including cryptococcal meningitis, and it is recommended to screen with a cryptococcal antigen test if the CD4 cell count is $<200$ cells $/ \mathrm{mm}^{3}$, and assess for danger signs of severe illness [14].

If patients are already receiving ART then this should be continued. All patients with active BU disease who are known or diagnosed as HIV positive but not on ART should initiate ART. This aims to reduce HIV associated mortality and morbidity, and HIV transmission [15]. Furthermore, as the immune system plays an important role in curing BU disease and in healing lesions, optimization of immunity with ART may be important to combat BU disease and potentially improve treatment outcomes (healing times, cure rates, long-term disability and recurrence rates). It has been found that in BU-HIV coinfected patients, a CD4 cell count $>500$ cells $/ \mathrm{mm}^{3}$ was associated with a reduction in the time needed to heal $\mathrm{BU}$ lesions by more than $50 \%$ compared to those with a CD4 count $\leq 500$ cells $/ \mathrm{mm}^{3}$ [2]. This suggests that healing times are significantly more prolonged among immune suppressed HIV positive individuals.

ART should begin as soon as possible after the start of BU treatment, preferably within 8 weeks, and as a priority in those with a CD4 cell count $<350$ cells $/ \mathrm{mm}^{3}$ or WHO stage 3 or 4 disease. Patients with advanced HIV are at immediate risk of further life-threatening opportunistic infections and delay in ART initiation may result in significant HIV-associated morbidity and mortality. As described earlier, this risk appears to be significant in BU-HIV co-infected patients, perhaps related to an increased risk of bacterial sepsis from secondarily infected BU lesions. Therefore, early reconstitution of immunity with ART in advanced BU disease may be important. Furthermore, this recommendation to start early also takes into account the fact that in routine programmes there may be delays in ART initiation whilst patients wait for assessment, training and availability of ART after completing their BU treatment. Also, as patients may receive BU treatment at a significant distance from ART centers, they may be lost to HIV care if ART initiation is delayed [16]. 
Fig. 2 A severe paradoxical reaction associated with a severe BU lesion on the left ankle of an HIV co-infected patient 2 weeks after commencing antiretroviral treatment

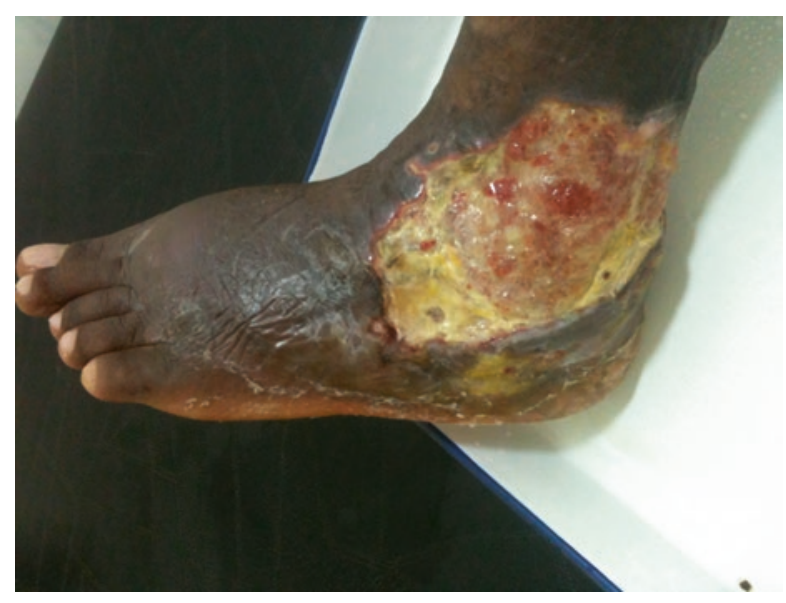

It is possible that early initiation of ART will lead to an increased incidence of paradoxical reactions associated with immune reconstitution when combining BU antibiotic treatment and ART $[1,17,18]$. This may lead to significant tissue damage and undesired consequences, especially if lesions are in sensitive areas (e.g. the face) (Fig. 2). Thus care needs to be taken when initiating ART. If severe paradoxical reactions occur ART should be continued and the use of prednisolone to minimize the severity of the effects should be considered $[17,19]$.

\subsection{HIV and BU Treatment Interactions}

There are a number of important issues regarding the use of antiretroviral drugs in patients receiving antibiotic treatment for BU. Firstly, as recommended for TB/HIV patients on ART using rifampicin containing regimens, the non-nucleoside reverse transcriptase inhibitor (NNRTI) component of the ART regimen should be efavirenz [20]. If this option is not available or appropriate then nevirapine can be used, but the lead-in dose of nevirapine should be omitted in the presence of rifampicin at the start of treatment. Additionally, caution should be exercised in the use of nevirapine particularly in patients with high or unknown CD4 cell-counts at initiation due to a potential increased risk of hypersensitivity and Stevens Johnson's syndrome [21]. Close monitoring during the initial weeks of therapy is recommended when nevirapine is initiated in these patients. Another alternative in adults and non-pregnant women is the integrase inhibitor dolutegravir which is preferred over efavirenz as a first-line antiretroviral in high-income settings and is being introduced in low- and middle-income settings. However, due to an interaction with rifampicin, it is currently recommended that the dose of dolutegravir should be doubled to $100 \mathrm{mg}$ daily.

There are concerns about significantly reduced levels of protease inhibitor (PI) medications and increased toxicity when they are used with rifampicin and therefore they are ideally avoided during BU antibiotic treatment. If the patient is already receiving a PI-based regimen, and they are NNRTI-naïve and not infected with the 
HIV-2 virus, the PI-based regimen should be changed to an NNRTI-based regimen using efavirenz. If they are not NNRTI-naïve or are infected with HIV-2 virus, then the recommended PI regimen to use is lopinavir/ritonavir (LPV/RTV) at either double dose ( $800 \mathrm{mg} / 200 \mathrm{mg}$ twice daily) or standard LPV dose with increased dose of RTV (400 mg/400 mg twice daily). However this combination in higher doses is frequently associated with high levels of toxicity and requires close clinical and laboratory monitoring. Again an alternative, if available, is for the integrase inhibitor dolutegravir to replace the PI during the 8-week BU treatment.

Efavirenz can reduce clarithromycin levels by up to $39 \%$ [22] which likely further compounds the known significant reduction of clarithromycin levels when coadministered with rifampicin [23]. Although the clinical consequences of these interactions are unknown, it could potentially lead to reduced effectiveness of the rifampicin/clarithromycin regimen for BU treatment, with secondary treatment failure and drug resistance. Increased toxicity is also reported when the two drugs are combined, with $46 \%$ of patients reported to develop a rash [24]. Therefore this combination should be used with caution. The alternative that avoids this interaction is rifampicin $10 \mathrm{mg} / \mathrm{kg}$ daily up to a maximum of $600 \mathrm{mg} /$ day plus moxifloxacin $400 \mathrm{mg}$ daily. This regimen has the added benefit of reducing the risk of rifampicin resistant TB if BU treatment is used in patients also infected with undetected drug sensitive active tuberculosis [25].

\subsection{Children}

All children should be commenced on ART as soon as possible within 8 weeks of the start of BU treatment. Efavirenz is not approved for clinical use in children $<3$ years of age. Therefore in this age-group if initiating ART whilst on BU treatment with rifampicin, nevirapine should be used instead of efavirenz at a dose of $200 \mathrm{mg} / \mathrm{m}^{2}$. An alternative is to use a triple NRTI ART regimen (AZT/3TC/ABC or AZT/3TC/ TDF). If already on a PI-based ART regimen when commencing BU treatment with rifampicin, LPV/RTV can be continued but the dose of ritonavir should be increased to achieve a 1:1 ratio with LPV. Alternative options include either replacing the LPV/ RTV with nevirapine at a dose of $200 \mathrm{mg} / \mathrm{m}^{2}$ or using a triple NRTI regimen.

\subsection{Tuberculosis}

All patients should be actively screened for tuberculosis (TB) before commencing BU treatment and before starting ART [26]. As most BU-HIV co-infected patients live in highly endemic areas for TB, there is a significant risk of TB co-infection. As HIV-infected patients have a higher risk of TB reactivation, especially when severely immunosuppressed, there is a risk of co-existent active TB disease. Therefore it is important to exclude active TB disease prior to commencing BU treatment, as BU treatment regimens are not adequate to treat active $\mathrm{TB}$, which may result in TB-related mortality and morbidity and the development of drug resistant TB. For 
those with symptoms suggestive of TB, this includes the use of Xpert MTB/RIF on sputum, and if CD4 count $\leq 100$ cells $/ \mathrm{mm}^{3}$ or the patient is seriously ill (at any CD4 cell count) the use of LF-LAM on urine. In those without symptoms of TB, TB preventative treatment should be started according to local guidelines [14].

\subsection{Service Provision}

BU patients found to be HIV-positive should be referred to clinicians trained in clinical management of HIV infection. Ideally, management should be integrated within the BU treatment centres to facilitate timely initiation of ART and avoid loss of patients to follow up which may occur during an external referral process for HIV care. If HIV management capacity in BU treatment centres is not possible, then referral to the nearest HIV treatment centre for care is recommended. Good cooperation between the BU and HIV treatment programmes at local, regional and national levels should be implemented to ensure the highest standard of care for BU-HIV co-infected patients. Approaches to support adherence to drug treatments for BU and HIV should be integrated, and programmes should implement a monitoring and reporting system to monitor and evaluate the outcomes of BU-HIV interventions.

\section{References}

1. Tuffour J, Bonney K, Owusu-Mireku E, Paintsil A, Pereko J, Yeboah-Manu D et al (2015) Challenges associated with management of Buruli ulcer/human immunodeficiency virus coinfection in a treatment center in Ghana: a case series study. Am J Trop Med Hyg 93(2):216-223

2. Christinet V, Comte E, Ciaffi L, Odermatt P, Serafini M, Antierens A, Rossel L, Nomo AB, Nkemenang P, Tsoungui A, Delhumeau C, Alexandra C (2014) Impact of human immunodeficiency virus on the severity of Buruli ulcer disease: results of a retrospective study in Cameroon. Open Forum Infect Dis 1(1):ofu021

3. Johnson RC, Nackers F, Glynn JR, de Biurrun BE, Zinsou C, Aguiar J et al (2008) Association of HIV infection and Mycobacterium ulcerans disease in Benin. AIDS 22(7):901-903

4. Vincent QB, Ardant MF, Marsollier L, Chauty A, Alcais A, Franco-Beninese Buruli Research Group (2014) HIV infection and Buruli ulcer in Africa. Lancet Infect Dis 14(9):796-797

5. Bayonne Manou LS, Portaels F, Eddyani M, Book AU, Vandelannoote K, De Jong BC (2013) Mycobacterium ulcerans disease (Buruli ulcer) in Gabon: 2005-2011. Me'decine et Sante' Tropicales 23:450-457

6. Raghunathan PL, Whitney EA, Asamoa K, Stienstra Y, Taylor TH Jr, Amofah GK et al (2005) Risk factors for Buruli ulcer disease (Mycobacterium ulcerans infection): results from a casecontrol study in Ghana. Clin Infect Dis 40(10):1445-1453

7. Kibadi K, Colebunders R, Muyembe-Tamfum JJ, Meyers WM, Portaels F (2010) Buruli ulcer lesions in HIV-positive patient. Emerg Infect Dis 16(4):738-739

8. Johnson RC, Ifebe D, Hans-Moevi A, Kestens L, Houessou R, Guedenon A et al (2002) Disseminated Mycobacterium ulcerans disease in an HIV-positive patient: a case study. AIDS 16(12):1704-1705

9. Bafende AE, Lukanu NP, Numbi AN (2002) Buruli ulcer in an AIDS patient. S Afr Med J 92(6):437

10. World Health Organisation (2014) Laboratory diagnosis of Buruli ulcer disease: a manual for health-care providers. WHO, Geneva 
11. World Health Organization (2014) Management of Buruli ulcer-HIV coinfection: technical update. WHO, Geneva

12. O'Brien DP, Ford N, Vitoria M, Christinet V, Comte E, Calmy A et al (2014) Management of BU-HIV co-infection. Tropical Med Int Health 19(9):1040-1047

13. World Health Organisation (2012) Treatment of Mycobacterium ulcerans disease (Buruli ulcer): guidance for health workers. WHO, Geneva

14. World Health Organization (2017) Guideline for managing advanced HIV disease and the timing for initiating antiretroviral therapy. WHO, Geneva

15. World Health Organization (2015) Guideline on when to start antiretroviral therapy and on preexposure prophylaxis for HIV. WHO, Geneva

16. Rosen S, Fox MP (2011) Retention in HIV care between testing and treatment in sub-Saharan Africa: a systematic review. PLoS Med 8(7):e1001056

17. Wanda F, Nkemenang P, Ehounou G, Tchaton M, Comte E, Toutous Trellu L et al (2014) Clinical features and management of a severe paradoxical reaction associated with combined treatment of Buruli ulcer and HIV co-infection. BMC Infect Dis 14(1):423

18. Komenan K, Elidje EJ, Ildevert GP, Yao KI, Kanga K, Kouame KA et al (2013) Multifocal Buruli ulcer associated with secondary infection in HIV positive patient. Case Rep Med 2013:348628

19. O'Brien DP, Jenkin G, Buntine J, Steffen CM, McDonald A, Horne S et al (2014) Treatment and prevention of Mycobacterium ulcerans infection (Buruli ulcer) in Australia: guideline update. Med J Aust 200(5):267-270

20. World Health Organization (2013) Consolidated guidelines on the use of antiretroviral drugs for treating and preventing HIV infection: recommendations for a public health approach. WHO, Geneva

21. Shubber Z, Calmy A, Andrieux-Meyer I, Vitoria M, Renaud-Thery F, Shaffer N et al (2013) Adverse events associated with nevirapine and efavirenz-based first-line antiretroviral therapy: a systematic review and meta-analysis. AIDS 27(9):1403-1412

22. Kuper JI, D'Aprile M (2000) Drug-drug interactions of clinical significance in the treatment of patients with Mycobacterium avium complex disease. Clin Pharmacokinet 39(3):203-214

23. Alffenaar JW, Nienhuis WA, de Velde F, Zuur AT, Wessels AM, Almeida D et al (2010) Pharmacokinetics of rifampin and clarithromycin in patients treated for Mycobacterium ulcerans infection. Antimicrob Agents Chemother 54(9):3878-3883

24. Bristol-Myers-Squibb (2010) Sustiva product information. Bristol-Myers-Squibb, Princetown

25. O'Brien DP, Comte E, Ford N, Christinet V, du Cros P (2013) Moxifloxacin for Buruli ulcer/ HIV coinfected patients: kill two birds with one stone? AIDS 27(14):2177-2179

26. World Health Organisation (2011) Guidelines for intensified tuberculosis case-finding and isoniazid preventive therapy for people living with HIV in resourceconstrained settings. WHO, Geneva

Open Access This chapter is licensed under the terms of the Creative Commons Attribution 4.0 International License (http://creativecommons.org/licenses/by/4.0/), which permits use, sharing, adaptation, distribution and reproduction in any medium or format, as long as you give appropriate credit to the original author(s) and the source, provide a link to the Creative Commons license and indicate if changes were made.

The images or other third party material in this chapter are included in the chapter's Creative Commons license, unless indicated otherwise in a credit line to the material. If material is not included in the chapter's Creative Commons license and your intended use is not permitted by statutory regulation or exceeds the permitted use, you will need to obtain permission directly from the copyright holder.

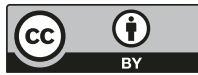

